IJMMS 29:10 (2002) 563-572

PII. S0161171202008086

http://ijmms.hindawi.com

(c) Hindawi Publishing Corp.

\title{
SOME EXTREMAL PROPERTIES OF SECTION SPACES OF BANACH BUNDLES AND THEIR DUALS
}

\author{
D. A. ROBBINS
}

Received 24 August 2001

\begin{abstract}
When $X$ is a compact Hausdorff space and $E$ is a real Banach space there is a considerable literature on extremal properties of the space $C(X, E)$ of continuous $E$-valued functions on $X$. What happens if the Banach spaces in which the functions on $X$ take their values vary over $X$ ? In this paper, we obtain some extremal results on the section space $\Gamma(\pi)$ and its dual $\Gamma(\pi)^{*}$ of a real Banach bundle $\pi: \mathscr{E} \rightarrow X$ (with possibly varying fibers), and point out the difficulties in arriving at totally satisfactory results.
\end{abstract}

2000 Mathematics Subject Classification: 46B20, 46E40, 46H25.

The present paper is motivated by the considerable literature which exists on various extremal properties of Banach spaces of the form $C(X, E)$, the continuous $E$-valued functions from the compact Hausdorff space $X$ to the Banach space $E$ with its norm topology. As examples of this literature, we cite for example [3, 6] and various of their references. Work has also been done on extremal properties of spaces of the form $C\left(X, E_{w}\right)$, where in this case $E_{w}$ is the Banach space $E$ with its weak topology (cf. [7]), and on $C\left(X, E_{w^{*}}^{*}\right)$, where $E_{w^{*}}^{*}$ is $E^{*}$ with its weak-* topology (see [12]).

The classic, and first, example of these extremal properties of $C(X, E)$ is the following. Recall that if $E$ is a Banach space and if $A \subset E$, then a point $z \in A$ is an extreme point of $A$ if and only if $z$ is not the midpoint of any line segment with endpoints in $A$. Denoting the set of extreme points of a set $A$ by extr(A), and the unit ball of a Banach space by $B(E)$, then a functional $\phi \in \operatorname{extr}\left(B\left(C(X, E)^{*}\right)\right)$ if and only if there exists some $f \in \operatorname{extr}\left(B\left(E^{*}\right)\right)$ and $x \in X$ such that $\phi=f \circ e v_{x}$, where $e v_{x}: C(X, E) \rightarrow E$, $e v_{x}(\sigma)=\sigma(x)$ is the evaluation map at $x \in X$. See [14]; a more general version can be found in [8].

For our purposes, $X$ denotes a compact Hausdorff space, and $\pi: \mathscr{E} \rightarrow X$ denotes a Banach bundle (i.e., bundle of Banach spaces) with real fibers $\left\{E_{x}: x \in X\right\}$; we assume throughout the paper that for all $x \in X, E_{x} \neq 0$. The total space $\mathscr{E}$ carries a topology such that the relative topology on each fiber $E_{x}$ is its original Banach space topology. We can regard $\mathscr{E}$ as the disjoint union $\bigcup\left\{E_{x}: x \in X\right\}$. (Alternatively, we can think of $\mathscr{E}$ as $\bigcup\left\{\{x\} \times E_{x}: x \in X\right\}$; this is the approach of [4], which uses fibered vector spaces.) See, for example, [4] or [10] for details on the construction of Banach bundles; the most important properties for our purposes are outlined here. We denote by $\Gamma(\pi)$ the space of sections (continuous choice functions $\sigma: X \rightarrow \mathscr{E}$ ) of the bundle $\pi: \mathscr{E} \rightarrow X ; \Gamma(\pi)$ is a Banach $C(X)$-module under the sup norm $\|\sigma\|=\sup \{\|\sigma(x)\|: x \in X\}$, pointwise addition, and the operation $(a \cdot \sigma)(x)=a(x) \sigma(x)(a \in C(X), x \in X)$. 
If $\pi: \mathscr{E} \rightarrow X$ is a Banach bundle, then the neighborhood system of a point $z_{0} \in E_{x} \subset \mathscr{E}$ can be described by tubes of the form $T=T(V, \sigma, \varepsilon)=\{z \in \mathscr{E}:\|z-\sigma(\pi(z))\|<$ $\varepsilon, \pi(z) \in V$ \}, where $V$ varies over the system of open neighborhoods of $x=\pi\left(z_{0}\right)$, $\varepsilon>0$ varies, and $\sigma \in \Gamma(\pi)$ is such that $\sigma\left(\pi\left(z_{0}\right)\right)=z_{0}$. If $z \in E_{x}$, then there exists $\sigma \in \Gamma(\pi)$ such that $\sigma(x)=z$ and $\|\sigma\|=\|z\|$. The function $x \mapsto\|\sigma(x)\|$ is upper semicontinuous from $X$ to $\mathbb{R}$ for each $\sigma \in \Gamma(\pi)$. If this function is continuous for each $\sigma \in \Gamma(\pi)$, we call $\pi: \mathscr{E} \rightarrow X$ a continuous bundle; in this case, the topology on $\mathscr{E}$ is Hausdorff. We call the bundle $\pi: \mathscr{E} \rightarrow X$ separable if there exists a countable set $\left\{\sigma_{n}\right\} \subset \Gamma(\pi)$ such that $\left\{\sigma_{n}(x)\right\}$ is dense in $E_{x}$ for each $x \in X$; this can be interpreted as a sort of uniform separability of the fibers $E_{x}$.

In thinking about the section space $\Gamma(\pi)$, perhaps the most important intuitive point to keep in mind is that when $\sigma \in \Gamma(\pi)$, then the values $\sigma(x) \in E_{x}$ vary continuously over (quite possibly very) different spaces as $x$ varies over $X$. A space of the form $C(X, E)$ can be regarded as the space of sections of the trivial bundle $\rho: \mathscr{T}=X \times$ $E \rightarrow X$, where $X \times E$ is given the product topology. Here, the total space $\mathscr{T}=X \times E$ can be thought of as a union of copies of $E$, and an element $\sigma \in C(X, E)$, which we usually think of as having values which vary continuously over the fixed set $E$, can be interpreted as a section in $\Gamma(\rho)$ which varies in a very nice way between copies of $E$.

To our knowledge, there is only one extreme point result for Banach bundles to be found in the literature: from [2, Theorem 2], reproven in [1], it can be shown that if $\pi: \mathscr{E} \rightarrow X$ is a Banach bundle, then $\phi \in \operatorname{extr}\left(B\left(\Gamma(\pi)^{*}\right)\right)$ if and only if there exists some $x \in X$ and $f_{x} \in \operatorname{extr}\left(B\left(E_{x}^{*}\right)\right)$ such that $\phi=f_{x} \circ e v_{x}$, where, again, $e v_{x}: \Gamma(\pi) \rightarrow E_{x}$, $\sigma \mapsto \sigma(x)$ is the evaluation map. This is clearly an analogue of the result for $C(X, E)$, and gives a reasonable first answer to the very loose question "what can we say about extreme points if the spaces, in which our continuous functions take their values, vary?"

With this as an introduction, and in pursuit of the question in the preceding paragraph, we now present some extremal results for section spaces of Banach bundles and their duals. All have analogues to those for spaces $C(X, E)$ and their duals. Because of the varying fibers in a Banach bundle, we have not been able to obtain results as fully descriptive as those for spaces $C(X, E)$; some of the difficulties will be pointed out as the exposition proceeds.

Our first results regard a section space $\Gamma(\pi)$ itself.

Proposition 1 (see [6, Theorem 1]). Let $\pi: \mathscr{E} \rightarrow X$ be a Banach bundle.

(1) Suppose that $\sigma \in \operatorname{extr}(B(\Gamma(\pi)))$. Then $\|\sigma(x)\|=1$ for all $x \in X$.

(2) Suppose, in addition, that $\pi: \mathscr{E} \rightarrow X$ is a Hausdorff bundle, that is, that $\mathscr{E}$ itself is a Hausdorff space. Let $\sigma \in B(\Gamma(\pi))$, with $\|\sigma\|=1$, and suppose that $K=\{x \in X$ : $\left.\sigma(x) \in \operatorname{extr}\left(B\left(E_{x}\right)\right)\right\}$ is dense in $X$. Then $\sigma \in \operatorname{extr}(B(\Gamma(\pi)))$. In particular, this is true if $\pi: \mathscr{E} \rightarrow X$ is a continuous bundle.

Proof. (1) Suppose that $\sigma \in B(\Gamma(\pi))$ and that for some $x \in X$ we have $\|\sigma(x)\|<1$. By upper semicontinuity of the norm, there exists an open neighborhood $V$ of $x$ such that for $y \in V,\|\sigma(y)\|<(1+\|\sigma(x)\|) / 2$. Choose $z \in E_{x}$ with $\|z\|=(1-\|\sigma(x)\|) / 2$, and a section $\tau \in \Gamma(\pi)$ such that $\tau(x)=z,\|\tau\|=\|z\|$, and such that $\tau(y)=0$ if $y \notin V$. Then $\sigma \pm \tau \neq \sigma$, and $\sigma=1 / 2((\sigma+\tau)+(\sigma-\tau))$. However, if $y \notin V$, we have 
$\|(\sigma \pm \tau)(y)\|=\|\sigma(y)\| \leq 1$; and, if $y \in V$, we have $\|(\sigma \pm \tau)(y)\| \leq\|\sigma(y)\|+\|\tau(y)\| \leq$ $(1+\|\sigma(x)\|) / 2+(1-\|\sigma(x)\|) / 2=1$. Thus, $\sigma \pm \tau \in B(\Gamma(\pi))$, and so $\sigma$ is not an extreme point of $B(\Gamma(\pi))$.

(2) Suppose that $\sigma=1 / 2\left(\tau+\tau^{\prime}\right)$, where $\tau, \tau^{\prime} \in B(\Gamma(\pi))$. By the hypothesis that $\sigma(x)$ be an extreme point in $B\left(E_{x}\right)$ whenever $x \in K$, it follows that for each $x \in K$ we have $\sigma(x)=\tau(x)=\tau^{\prime}(x)$. Suppose now that for some $y \in X$ we have $\tau(y) \neq$ $\tau^{\prime}(y)$. Because $\pi: \mathscr{E} \rightarrow X$ is a Hausdorff bundle, there are disjoint tubes around $\tau(y)$ and $\tau^{\prime}(y)$. In particular, for some neighborhood $V$ of $y$ and some $\varepsilon>0$, we have $\left\|\tau(x)-\tau^{\prime}(x)\right\| \geq \varepsilon$ for all $x \in V$. But by the density of $K$, this is impossible. The second assertion follows because a continuous bundle is Hausdorff.

Recall that a point $z \in B(E)$ is called a sequential point of continuity if whenever $\left\{z_{n}\right\} \subset B(E)$ is a sequence which converges weakly to $z$, then $\left\|z_{n}-z\right\| \rightarrow 0$. In [3, Theorem 3] it is shown that $B(C(X, E))$ contains no weak sequential points of continuity. The same is true for a large class of bundles.

Proposition 2. Let $\pi: \mathscr{E} \rightarrow X$ be a continuous and separable bundle, and suppose that $X$ is infinite. Then $B(\Gamma(\pi))$ has no sequential points of continuity.

Proof. We follow the outline of [3, Theorem 3], with an adjustment to take care of the bundle situation.

Let $\sigma \in B(\Gamma(\pi))$, and let $U=\{x:\|\sigma(x)\|<2 / 3\}$ and $V=\{x:\|\sigma(x)\|>1 / 3\}$. By continuity of the norm, both $U$ and $V$ are open, and their union is $X$. At least one of $U$ or $V$ is infinite, say $U$ for the moment. We can find open, pairwise disjoint, nonempty $U_{n} \subset U$; choose $x_{n} \in U_{n}$. Choose $z_{n} \in E_{x_{n}}$ such that $\left\|z_{n}\right\|=1 / 3$, and choose $\sigma_{n} \in \Gamma(\pi)$ such that $\sigma_{n}\left(x_{n}\right)=z_{n}$ and $\left\|\sigma_{n}\right\|=1 / 3$. Choose continuous functions $a_{n}$ : $X \rightarrow[0,1]$ such that $a_{n}\left(x_{n}\right)=1$ and such that $a_{n}\left(X \backslash U_{n}\right)=0$. Let $\tau_{n}=\sigma+a_{n} \sigma_{n}$. Then $\left\|\tau_{n}(y)\right\|=\left\|\sigma(y)+a_{n}(y) \sigma_{n}(y)\right\|=\|\sigma(y)\| \leq 1$, if $y \notin U_{n}$. On the other hand, if $y \in U_{n}$, then $\left\|\tau_{n}(y)\right\| \leq\|\sigma(y)\|+a_{n}(y) * 1 / 3 \leq 1$, so that $\tau_{n} \in B(\Gamma(\pi))$. Evidently, $\left\|\tau_{n}-\sigma\right\|=\left\|a_{n} \sigma_{n}\right\|=1 / 3$.

Now, let $\phi \in \Gamma(\pi)^{*}$. By [5], there exists $\mu \geq 0 \in M(X)$ and a selection $\eta: X \rightarrow \dot{\bigcup}\left\{E_{x}^{*}\right.$ : $x \in X\}$, the disjoint union of the $E_{x}^{*}$, such that (a) for all $\sigma \in \Gamma(\pi), x \mapsto\langle\sigma(x), \eta(x)\rangle$ is Borel measurable; (b) $\|\eta(x)\|=1 \mu$-a.e.; and (c) $\langle\sigma, \phi\rangle=\int_{X}\langle\sigma(x), \eta(x)\rangle d \mu$. Then

$$
\begin{aligned}
\left|\left\langle\tau_{n}-\sigma, \phi\right\rangle\right| & =\left|\int_{X}\left\langle\tau_{n}(x)-\sigma(x), \eta(x)\right\rangle d \mu\right| \\
& =\left|\int_{X}\left\langle a_{n}(x) \sigma_{n}(x), \eta(x)\right\rangle d \mu\right| \\
& \leq \int_{U_{n}}|| a_{n}(x) \sigma_{n}(x) \| d \mu \\
& \leq \frac{1}{3} \mu\left(U_{n}\right) \longrightarrow 0 .
\end{aligned}
$$

Thus, $\sigma$ is not a sequential point of continuity.

Similarly, if $V$ above is infinite, choose open, pairwise disjoint, nonempty $V_{n} \subset$ $V, x_{n} \in V_{n}$, and continuous functions $a_{n}: X \rightarrow[0,1]$, such that $a_{n}\left(x_{n}\right)=1$ and $a_{n}\left(X \backslash V_{n}\right)=0$. Set $\sigma_{n}=\left(1-a_{n}\right) \sigma$. Clearly, $\left\|\sigma_{n}\right\| \leq\|\sigma\| \leq 1$ and $\left\|\sigma_{n}-\sigma\right\| \geq \| \sigma_{n}\left(x_{n}\right)-$ 
$\sigma\left(x_{n}\right)\|=\| \sigma\left(x_{n}\right) \|>1 / 3$. We again have

$$
\begin{aligned}
\left|\left\langle\tau_{n}-\sigma, \phi\right\rangle\right| & =\left|\int_{X}\left\langle\sigma_{n}(x)-\sigma(x), \eta(x)\right\rangle d \mu\right| \\
& =\left|\int_{V_{n}}\left\langle a_{n} \sigma_{n}(x), \eta(x)\right\rangle d \mu\right| \\
& \leq \int_{V_{n}}\left\|\sigma_{n}(x)\right\| d \mu \\
& \leq \mu\left(V_{n}\right) \longrightarrow 0 .
\end{aligned}
$$

In this case also, $\sigma$ is not a sequential point of continuity.

Recall that a point $z \in B(E)$ is called a strongly extreme point for each $\varepsilon>0$ there exists $\delta>0$ such that if $\|z \pm y\| \leq 1+\delta$, then $\|y\| \leq \varepsilon$. It is shown in [3] that a function $\sigma \in B(C(X, E))$ is a strongly extreme point if and only if $\sigma(x)$ is a strongly extreme point of $B(E)$ for each $x \in X$. We can do somewhat better in the bundle case in one direction.

Recall (see [4] or [10]) that if $C \subset X$ is a closed set, then there is a bundle $\pi_{C}: \mathscr{E}_{C} \rightarrow C$, called the restriction of $\pi$ to $C$, where $\mathscr{E}_{C}=\mathscr{E} \cap \pi^{-1}(C)$. We have $\Gamma\left(\pi_{C}\right) \simeq \Gamma(\pi) / I_{C} \Gamma(\pi)$, where $I_{C}=\{a \in C(X): a(x)=0$ for all $x \in C\}$ and $I_{C} \Gamma(\pi)$ is the closed span in $\Gamma(\pi)$ of $\left\{a \cdot \sigma: a \in I_{C}, \sigma \in \Gamma(\pi)\right\}$. Write $I_{x}$ for $I_{\{x\}}$. An element $\tau_{C} \in \Gamma\left(\pi_{C}\right)$ if and only if $\tau_{C}$ is the restriction to $C$ of some $\tau \in \Gamma(\pi)$. In particular, $E_{x} \simeq \Gamma\left(\pi_{\{x\}}\right) \simeq \Gamma(\pi) / I_{x} \Gamma(\pi)$.

Proposition 3. Let $\pi: \mathscr{E} \rightarrow X$ be a bundle. If $\sigma \in B(\Gamma(\pi))$ is a strongly extreme point, then $\sigma_{C}=$ the restriction of $\sigma$ to $C$ is a strongly extreme point in $\Gamma\left(\pi_{C}\right)$ for each closed $C \subset X$. In particular, for each $x \in X, \sigma(x)$ is a strongly extreme point in the unit ball of $E_{x}$ (and, by the above discussion, $E_{x} \simeq \Gamma\left(\pi_{\{x\}}\right)$ ).

Proof. We use the Tietze extension theorem for bundles; see [9]. Let $\sigma \in B(\Gamma(\pi))$ be a strongly extreme point, let $C \subset X$ be closed, and let $\varepsilon>0$ be given. Choose $\delta>0$ such that if $\|\sigma \pm \omega\| \leq 1+\delta$ then $\|\omega\| \leq \varepsilon$. Suppose that $\tau_{C} \in \Gamma\left(\pi_{C}\right)$ is such that $\left\|\sigma_{C} \pm \tau_{C}\right\|<1+\delta / 2$. There exists $\tau \in \Gamma(\pi)$ such that the restriction of $\tau$ to $C$ is $\tau_{C}$ and such that $\|\boldsymbol{\tau}\|=\left\|\boldsymbol{\tau}_{C}\right\|$. Clearly, then, $\sigma \pm \boldsymbol{\tau}$ extends $\sigma_{C} \pm \boldsymbol{\tau}_{C}$. By the upper semicontinuity of the norm, there exists a neighborhood $V$ of $C$ such that if $y \in V$ then $\|(\sigma \pm \tau)(y)\|<1+\delta$. Choose a continuous function $i_{V}: X \rightarrow[0,1]$ such that $i_{V}(C)=0$ and $i_{V}(X \backslash V)=1$. Then $\left\|\sigma \pm\left(1-i_{V}\right) \tau\right\|<1+\delta$, and $\left(1-i_{V}\right) \tau$ is an extension of $\tau_{C}$. By the choice of $\delta$, we have $\left\|\tau_{C}\right\| \leq\left\|\left(1-i_{V}\right) \tau\right\|<\varepsilon$.

The converse to this result is demonstrated in [3] for the case of $C(X, E)$. With the next two propositions, we provide a condition which will give us a converse for the case of section spaces, and show that the condition actually obtains in the case of $C(X, E)$, thus giving an alternative proof of the second part of [3, Theorem 2].

Note that if $\sigma \in \Gamma(\pi)$ and $\sigma(x)$ is a strongly extreme point of $B\left(E_{x}\right)$ for each $x \in X$, then given $\varepsilon>0$ there is some $\delta(x, \varepsilon, \sigma)>0$ such that if $\|\sigma(x) \pm z\| \leq 1+\delta(x, \varepsilon, \sigma)$, then $\|z\| \leq \varepsilon$. For a fixed $x, \varepsilon, \sigma$, let $\delta_{0}(x, \varepsilon, \sigma)=\sup \{\delta(x, \varepsilon, \sigma):$ if $\|\sigma(x) \pm z\| \leq$ $1+\delta(x, \varepsilon, \sigma)$ then $\|z\| \leq \varepsilon\}$. Clearly, $\delta_{0}(x, \varepsilon, \sigma)>0$, and if $\|\sigma(x) \pm z\| \leq 1+\delta_{0}(x, \varepsilon, \sigma)$, then $\|z\| \leq \varepsilon$. 
Proposition 4. Suppose that $\sigma \in \Gamma(\pi)$ and that $\sigma(x)$ is a strongly extreme point of $B\left(E_{x}\right)$ for each $x \in X$. Suppose also that the function $x \mapsto \delta_{0}(x, \varepsilon, \sigma)$ is bounded away from 0 for each $\varepsilon>0$. Then $\sigma$ is a strongly extreme point of $B(\Gamma(\pi))$. In particular, if $x \mapsto \delta_{0}(x, \varepsilon, \sigma)$ is lower semicontinuous on $X$, then $\sigma$ is a strongly extreme point of $B(\Gamma(\pi))$.

Proof. Fix $\varepsilon>0$. Let $\delta_{0}=\delta_{0}(\varepsilon, \sigma)=\inf \left\{\delta_{0}(x, \varepsilon, \sigma): x \in X\right\}>0$. Suppose that $\tau \in \Gamma(\pi)$ is such that $\|\sigma \pm \tau\| \leq 1+\delta_{0}$. Then, given $x \in X$, we have $\|\sigma(x) \pm \tau(x)\| \leq$ $1+\delta_{0} \leq 1+\delta_{0}(x, \varepsilon, \sigma)$, so that $\|\tau(x)\| \leq \varepsilon$. Hence $\|\tau\| \leq \varepsilon$.

Proposition 5. Let $\sigma \in C(X, E)$, and suppose that $\sigma(x)$ is a strongly extreme point of $B\left(E_{x}\right)$ for each $x \in X$. Then $x \mapsto \delta_{0}(x, \varepsilon, \sigma)$ is lower semicontinuous for each $\varepsilon>0$.

Proof. Fix $x \in X$. By the continuity of $\sigma$, for each $k \in \mathbb{N}, k \geq 2$, there exists a neighborhood $V_{k}$ of $x$ such that if $y \in V_{k}$, then $\|\sigma(x)-\sigma(y)\|<\delta_{0}(x, \varepsilon, \sigma) / k$. Suppose that $y \in V_{k}$, and that $\|\sigma(y) \pm z\| \leq 1+((k-1) / k) \delta_{0}(x, \varepsilon, \sigma)$. Then

$$
\begin{aligned}
\|\sigma(x) \pm z\| & \leq\|\sigma(x)-\sigma(y)\|+\|\sigma(y) \pm z\| \\
& <\frac{\delta_{0}(x, \varepsilon, \sigma)}{k}+1+\frac{k-1}{k} \delta_{0}(x, \varepsilon, \sigma) \\
& =1+\delta_{0}(x, \varepsilon, \sigma),
\end{aligned}
$$

so that $\|z\|<\varepsilon$. Thus, when $y \in V_{k}, \delta_{0}(y, \varepsilon, \sigma)>((k-1) / k) \delta_{0}(x, \varepsilon, \sigma)$, so that $x \mapsto$ $\delta_{0}(x, \varepsilon, \sigma)$ is lower semicontinuous.

We now consider extreme point theorems in $B\left(\Gamma(\pi)^{*}\right)$. Recall that for a bundle $\pi$ : $\mathscr{E} \rightarrow X$, there is a canonical isometric injection $j_{x}: E_{x}^{*} \rightarrow \Gamma(\pi)^{*}$, given by $\left\langle\sigma, j_{x}(f)\right\rangle=$ $\langle\sigma(x), f\rangle$; that is, $j_{x}(f)=f \circ e v_{x}$. Conversely, it can be shown that there is a surjective $L$-projection $P_{x}: \Gamma(\pi)^{*} \rightarrow j_{x}\left(E_{x}^{*}\right)$ given by $P_{x}(\phi)=\phi_{x}$, where $\phi_{x}=$ weak-* $\lim _{V}((1-$ $\left.i_{V}\right) \phi$ ), where $V \in \mathscr{V}$, the system of open neighborhoods of $x \in X$, and, for each $V \in \mathscr{V}$, the continuous function $i_{V}: X \rightarrow[0,1], i_{V}(x)=0$ and $i_{V}(X \backslash V)=0$ (see $\left.[4,11]\right)$. Then for $f \in E_{x}^{*}$, we have $P_{x}\left(j_{x}(f)\right)=j_{x}(f)$, and $P_{x}$ is an isometry on $j_{x}\left(E_{x}^{*}\right)$. Using the notation preceding Proposition 3, the range of $P_{x}$ can also be expressed as $\left(I_{x} \Gamma(\pi)\right)^{\perp}$; this is an $L$-summand in $\Gamma(\pi)^{*}$.

Proposition 6. Let $\pi: \mathscr{E} \rightarrow X$ be a bundle, and suppose that $f \in B\left(E_{x}^{*}\right)$ is a strongly extreme point. Then $j_{x}(f)=f \circ e v_{x}$ is a strongly extreme point in $B\left(\Gamma(\pi)^{*}\right)$. Conversely, if $\phi \in B\left(\Gamma(\pi)^{*}\right)$ is a strongly extreme point, then there exist $x \in X$ and a strongly extreme point $f \in B\left(E_{x}^{*}\right)$ such that $\phi=j_{x}(f)=f \circ e v_{x}$.

Proof. We use [13, Lemma 3]: let $P$ be an $L$-projection in a Banach space $E$, and suppose that $M=\operatorname{range}(P)$ and $N=\operatorname{ker}(P)$. Then $z \in B(E)$ is a strongly extreme point if and only if $z \in B(M)$ or $z \in B(N)$ and is a strongly extreme point of that set. Suppose now that $\phi \in B\left(\Gamma(\pi)^{*}\right)$ is a strongly extreme point. Then, in particular, $\phi$ is an extreme point, and so there exists an $x \in X$ and $f \in B\left(E_{x}^{*}\right)$ such that $\phi=j_{x}(f)=f \circ e v_{x}$. Since $P_{x}(\phi)=j_{x}(f) \in j_{x}\left(E_{x}^{*}\right)=\operatorname{range}\left(P_{x}\right)$, and since $P_{x}$ is an $L$-projection, it follows by the above that $j_{x}(f)$ is a strongly extreme point in $j_{x}\left(B\left(E_{x}^{*}\right)\right)$. Since $j_{x}$ is an isometric isomorphism, $f$ is then a strongly extreme point in $B\left(E_{x}^{*}\right)$. Conversely, if $f$ is a strongly extreme point in $B\left(E_{x}^{*}\right)$, then $j_{x}(f)$ is a strongly extreme point in $j_{x}\left(B\left(E_{x}^{*}\right)\right)$. Again 
applying Rao's lemma, we see that $j_{x}(f)=f \circ e v_{x}$ is a strongly extreme point in $B\left(\Gamma(\pi)^{*}\right)$.

Recall that a point $f \in E^{*},\|f\|=1$, is a point of weak-* continuity of $B\left(E^{*}\right)$ if and only if whenever we have a net $f_{\alpha} \rightarrow f$ weak-*, with $\left\|f_{\alpha}\right\| \leq 1$, then $\left\|f_{\alpha}-f\right\| \rightarrow 0$. If $\pi: \mathscr{E} \rightarrow X$ is a bundle, then for $\phi \in \Gamma(\pi)^{*}$ and $x \in X$, write $\phi_{x}=P_{x}(\phi)$, where $P_{x}$ is the $L$-projection described earlier. We can find some of the weak-* points of continuity $B\left(\Gamma(\pi)^{*}\right)$ by using techniques of [6].

We first make the following observation: let $x \in X$ be isolated, and let $z \in E_{x}$. Then the selection $\sigma$ on $X$ defined by

$$
\sigma(y)= \begin{cases}z, & \text { if } x=y, \\ 0, & \text { if } x \neq y\end{cases}
$$

is an element of $\Gamma(\pi)$. Moreover, if $\phi \in \Gamma(\pi)^{*}$, we have

$$
\left\langle\sigma, \phi-\phi_{x}\right\rangle=\lim _{V}\left\langle\sigma, \phi-\left(1-i_{V}\right) \phi\right\rangle=\lim _{V}\left\langle i_{V} \sigma, \phi\right\rangle=0,
$$

as $V$ ranges over the neighborhood system of $x$, since the function $i_{V}$ which is 1 at $x$ and 0 elsewhere is an identity for the maximal ideal $I_{x} \subset C(X)$. Since $\phi_{x} \in\left(I_{x} \Gamma(\pi)\right)^{\perp} \simeq$ $E_{x}^{*}$, we may write $\phi_{x}=f_{x} \circ e v_{x}$ for some $f_{x} \in E_{x}^{*}$. Thus, $\left\langle\sigma, \phi_{x}\right\rangle=\left\langle\sigma(x), f_{x}\right\rangle$.

Proposition 7. Let $\pi: \mathscr{E} \rightarrow X$ be a bundle, and suppose that $\phi \in \Gamma(\pi)^{*}$ has the form $\phi=\sum_{x \in I} \phi_{x}$, where $I=\{x \in X: x$ is isolated $\}$. Suppose further that for each $x \in I$ either $f_{x}=0$ or $f_{x} /\left\|f_{x}\right\|$ is a weak-* point of continuity of $B\left(E_{x}^{*}\right)$ and that $\sum_{x \in I}\left\|f_{x}\right\|=\sum_{x \in I}\left\|\phi_{x}\right\|=1$, where $\phi_{x}=f_{x} \circ$ ev $v_{x}$. Then $\phi$ is a weak-* point of continuity of $B\left(\Gamma(\pi)^{*}\right)$.

Proof. It can be easily shown that $\sum_{x \in I} P_{x}$ is an $L$-projection, and so it follows that $\|\phi\|=\sum_{x \in I}\left\|\phi_{x}\right\|=\sum_{x \in I_{0}}\left\|\phi_{x}\right\|$, where $I_{0}=\left\{x \in I: \phi_{x} \neq 0\right\}$.

Let $x \in I_{0}$ be arbitrary. If $\phi_{\alpha} \rightarrow \phi$ weak-* in $\Gamma(\pi)^{*}$, with $\left\|\phi_{\alpha}\right\| \leq 1$ and if $z \in E_{x}$, then a selection of the form $\sigma(y)=z$, if $x=y$, and $\sigma(y)=0$ otherwise, is an element of $\Gamma(\pi)$. From the preceding observation, we have

$$
\left\langle\sigma,\left(\phi_{\alpha}\right)_{x}\right\rangle=\left\langle\sigma(x),\left(f_{\alpha}\right)_{x}\right\rangle=\left\langle\sigma, \phi_{\alpha}\right\rangle \longrightarrow\left\langle\sigma, \phi_{x}\right\rangle=\langle\sigma, \phi\rangle=\left\langle\sigma(x), f_{x}\right\rangle,
$$

and so $\left(f_{\alpha}\right)_{x} \rightarrow f_{x}$ weak-* in $E_{x}^{*}$. Since the norm is lower semicontinuous with respect to the weak-* topology, we obtain liminf $\left\|\left(f_{\alpha}\right)_{x}\right\| \geq\left\|f_{x}\right\|$, and hence find that $\liminf \left\|\left(\phi_{\alpha}\right)_{x}\right\| \geq\left\|\phi_{x}\right\|$.

We now have

$$
\begin{aligned}
1 & =\|\phi\|=\left\|\sum_{x \in I} \phi_{x}\right\|=\sum_{x \in I_{0}}\left\|\phi_{x}\right\| \\
& \leq \sum_{x \in I_{0}} \liminf \left\|\left(\phi_{\alpha}\right)_{x}\right\| \leq \liminf \sum_{x \in I_{0}}\left\|\left(\phi_{\alpha}\right)_{x}\right\| \\
& =\liminf \left\|\phi_{\alpha}\right\| \leq 1 .
\end{aligned}
$$

If for some $x \in I_{0}$ we have liminf $\left\|\left(\phi_{\alpha}\right)_{x}\right\|>\left\|\phi_{x}\right\|$, then from the above we are led to the contradiction $1<1$. Hence, liminf $\left\|\left(\phi_{\alpha}\right)_{x}\right\|=\left\|\phi_{x}\right\|$ for all $x \in I_{0}$. A similar remark shows that limsup $\left\|\left(\phi_{\alpha}\right)_{x}\right\|=\left\|\phi_{x}\right\|$, and so we have $\lim \left\|\left(\phi_{\alpha}\right)_{x}\right\|=\left\|\phi_{x}\right\|$ for all $x \in I_{0}$. 
Now, by assumption, $f_{x} /\left\|f_{x}\right\|$ is a weak-* point of continuity of $B\left(E_{x}^{*}\right)$ for each $x \in I_{0}$. Since $\left(f_{\alpha}\right)_{x} /\left\|\left(f_{\alpha}\right)_{x}\right\| \rightarrow f_{x} /\left\|f_{x}\right\|$ weak-*, we then have

$$
\left\|\frac{\left(f_{\alpha}\right)_{x}}{\left\|\left(f_{\alpha}\right)_{x}\right\|}-\frac{f_{x}}{\left\|f_{x}\right\|}\right\| \rightarrow 0,
$$

and thus $\left\|\left(f_{\alpha}\right)_{x}-f_{x}\right\|=\left\|\left(\phi_{\alpha}\right)_{x}-\phi_{x}\right\| \rightarrow 0$ for each $x \in I_{0}$.

Finally, for $\varepsilon>0$, we can choose a finite $I^{\prime} \subset I_{0}$ such that $\sum_{x \in I^{\prime}}\left\|\phi_{x}\right\|=\sum_{x \in I^{\prime}}\left\|f_{x}\right\|>$ $1-\varepsilon$. When $\alpha$ is sufficiently large, $\sum_{x \in I^{\prime}}\left\|\left(f_{\alpha}\right)_{x}-f_{x}\right\|=\sum_{x \in I^{\prime}}\left\|\left(\phi_{\alpha}\right)_{x}-\phi_{x}\right\|<\varepsilon$. Then for all these large $\alpha$ we have

$$
\begin{aligned}
\left\|\phi_{\alpha}-\phi\right\| & =\sum_{x \in I^{\prime}}\left\|\left(\phi_{\alpha}\right)_{x}-\phi_{x}\right\|+\left\|\left(\phi_{\alpha}-\phi\right)-\left(\sum_{x \in I^{\prime}}\left(\phi_{\alpha}\right)_{x}-\phi_{x}\right)\right\| \\
& <\varepsilon+\left\|\phi_{\alpha}-\sum_{x \in I^{\prime}}\left(\phi_{\alpha}\right)_{x}\right\|+\left\|\phi-\sum_{x \in I^{\prime}} \phi_{x}\right\| \\
& =\varepsilon+\left\|\phi_{\alpha}\right\|-\left\|\sum_{x \in I^{\prime}}\left(\phi_{\alpha}\right)_{x}\right\|+\|\phi\|-\left\|\sum_{x \in I^{\prime}} \phi_{x}\right\| \\
& \leq \varepsilon+2+\sum_{x \in I^{\prime}}\left\|\left(\phi_{\alpha}\right)_{x}-\phi_{x}\right\|-2 \sum_{x \in I^{\prime}}\left\|\phi_{x}\right\| \\
& <\varepsilon+2+\varepsilon+2(1-\varepsilon) \\
& =4 \varepsilon .
\end{aligned}
$$

Thus, $\phi_{\alpha} \rightarrow \phi$ in norm, and so $\phi$ is a weak-* point of continuity. Clearly, this proof owes its details to [6, Theorem 6].

It is shown in [6] that the condition analogous to the above for $C(X, E)^{*}$ actually characterizes weak-* points of continuity in $B\left(C(X, E)^{*}\right)$. The proof in this case uses very strongly the description of $C(X, E)^{*}$. However, even in the case of a continuous and separable bundle, where there is at least some description of $\Gamma(\pi)^{*}$, it is not clear how to arrive at the whole converse to Proposition 7 . We do obtain a rather unsatisfactory partial result for bundles.

Proposition 8. Let $\pi: \mathscr{E} \rightarrow X$ be a bundle, and suppose that $\phi \in B\left(\Gamma(\pi)^{*}\right),\|\phi\|=1$, is a weak-* point of continuity. Suppose that for a given $x \in X, \phi_{x}=f_{x} \circ$ e $v_{x} \neq 0$. Then $f_{x} /\left\|f_{x}\right\|$ is a weak-* point of continuity of $B\left(E_{x}^{*}\right)$, and $\phi_{x} /\left\|\phi_{x}\right\|$ is a weak-* point of continuity of $B\left(\Gamma(\pi)^{*}\right)$.

Proof. Let $\left\{g_{\alpha}\right\}$ be a net in $B\left(E_{x}^{*}\right)$ such that $g_{\alpha} \rightarrow f_{x} /\left\|f_{x}\right\|$ weak-*. Let $\phi_{\alpha}=$ $\phi-\phi_{x}+j_{x}\left(\left\|f_{x}\right\| g_{\alpha}\right)$. Then $\phi_{a} \rightarrow \phi$ weak-* in $\Gamma(\pi)^{*}$, and since

$$
\begin{aligned}
\left\|\phi_{\alpha}\right\| & =\left\|\phi-\phi_{x}+j_{x}\left(\left\|f_{x}\right\| g_{\alpha}\right)\right\| \\
& \leq\left\|\phi-\phi_{x}\right\|+\left\|j_{x}\left(\left\|f_{x}\right\| g_{\alpha}\right)\right\| \\
& \leq\left\|\phi-\phi_{x}\right\|+\left\|f_{x}\right\| \\
& =\left\|\phi-\phi_{x}\right\|+\left\|\phi_{x}\right\| \\
& =\|\phi\|,
\end{aligned}
$$


it follows that $\left\|\phi_{\alpha}-\phi\right\|=\left\|\phi_{x}-j_{x}\left(\left\|f_{x}\right\| g_{\alpha}\right)\right\|=\left\|f_{x}-\right\| f_{x}\left\|g_{\alpha}\right\| \rightarrow 0$. Thus, $f_{x}$ is a weak-* point of continuity of $B\left(E_{x}^{*}\right)$.

Now, suppose that $\left\{\psi_{\alpha}\right\}$ is a net in $B\left(\Gamma(\pi)^{*}\right)$ such that $\psi_{\alpha} \rightarrow \phi_{x} /\left\|\phi_{x}\right\|$ weak-*. We then have $\phi-\phi_{x}+\left\|\phi_{x}\right\| \psi_{\alpha} \rightarrow \phi$ weak-*, and

$$
\left\|\phi-\phi_{x}+\right\| \phi_{x}\left\|\psi_{\alpha}\right\| \leq\left\|\phi-\phi_{x}\right\|+\|\| \phi_{x}\left\|\psi_{\alpha}\right\| \leq 1,
$$

so that $\left\|\phi-\phi_{x}+\right\| \phi_{x}\left\|\psi_{\alpha}-\phi\right\|=\|\| \phi_{x}\left\|\psi_{\alpha}-\phi_{x}\right\| \rightarrow 0$, and thus $\phi_{x} /\left\|\phi_{x}\right\|$ is a weak-* point of continuity of $B\left(\Gamma(\pi)^{*}\right)$.

In further analogy with [6], we can identify some points of continuity and sequential points of continuity in $B\left(\Gamma(\pi)^{*}\right)$; see [6, Proposition 11]. The difficulties in a complete characterization of points of continuity in $B\left(C(X, E)^{*}\right)$ which are discussed there are even less tractable for $B\left(\Gamma(\pi)^{*}\right)$.

Proposition 9. Let $\pi: E \rightarrow X$ be a bundle, and suppose that $\phi \in B\left(\Gamma(\pi)^{*}\right)$ has the form $\phi=\sum_{x \in X} f_{x} \circ e v_{x}=\sum_{x \in X} \phi_{x}$, where for each $x \in X$ either $f_{x}=0$ or $f_{x} /\left\|f_{x}\right\|$ is a point of continuity (sequential point of continuity) of $B\left(E_{x}^{*}\right)$, and $\|\phi\|=1=$ $\sum_{x \in X}\left\|f_{x} \circ e v_{x}\right\|$. Then $\phi$ is a point of continuity (sequential point of continuity) of $B\left(\Gamma(\pi)^{*}\right)$.

Proof. If $x \in X$ and $\phi_{x}^{* *} \in E_{x}^{* *}$, define $\Phi_{x}^{* *} \in \Gamma(\pi)^{* *}$ by $\left\langle\phi, \Phi_{x}^{* *}\right\rangle=\left\langle j_{x}^{-1}\left(\phi_{x}\right), \phi_{x}^{* *}\right\rangle$ for each $\phi \in \Gamma(\pi)^{*}$, where $\phi_{x}$ and $j_{x}$ are as described earlier. If $\phi \in \Gamma(\pi)^{*}$, and if $\phi_{\alpha} \rightarrow \phi$ weakly in $\Gamma(\pi)^{*}$, then for each $x \in X$ we have

$$
\left\langle\phi_{\alpha}, \Phi_{x}^{* *}\right\rangle=\left\langle j_{x}^{-1}\left(\left(\phi_{\alpha}\right)_{x}\right), \phi_{x}^{* *}\right\rangle \longrightarrow\left\langle\phi, \Phi_{x}^{* *}\right\rangle=\left\langle j_{x}^{-1}\left(\phi_{x}\right), \phi_{x}^{* *}\right\rangle,
$$

so that $j_{x}^{-1}\left(\left(\phi_{\alpha}\right)_{x}\right) \rightarrow j_{x}^{-1}\left(\phi_{x}\right)$ weakly in $E_{x}^{*}$. Hence, if $\phi$ is of the form described in the statement of the proposition and $\phi_{\alpha} \rightarrow \phi$ weakly in $\Gamma(\pi)^{*}$, we have $\| j_{x}^{-1}\left(\left(\phi_{\alpha}\right)_{x}\right)-$ $j_{x}^{-1}\left(\phi_{x}\right)\|=\|\left(\phi_{\alpha}\right)_{x}-\phi_{x} \| \rightarrow 0$. By repeating the argument at the end of Proposition 7 , we obtain that $\left\|\phi_{\alpha}-\phi\right\| \rightarrow 0$. The case of sequential continuity is clearly similar.

EXAMPLE 10. It is well known that for a Banach space $E$, we have $C(X, E)^{*} \simeq M\left(X, E^{*}\right)$, the space of $E^{*}$-valued countably additive Borel measures of bounded variation on $X$, equipped with the variation norm. In [6] it is shown that if $\mu \in B\left(C(X, E)^{*}\right)$ is a point of weak-* continuity, then $\mu(\{x\})=0$ whenever $x$ is non-isolated. The analogous result for $B\left(\Gamma(\pi)^{*}\right)$ then takes this form: whenever $\phi \in B\left(\Gamma(\pi)^{*}\right)$ and $\phi$ is a weak-* point of continuity, and $x \in X$ is isolated, then $\phi_{x}=0$. In general, this is not true. Let $X=[0,1]$, and let $X_{d}$ be $X$ with its discrete topology. We can regard $c_{0}\left(X_{d}\right)$, the closure under the sup-norm of the set of (real-valued) functions on $X$ with finite support, as the space $\Gamma(\pi)$ of sections of a bundle $\pi: \mathscr{E} \rightarrow X$; this is referred to in [10] as the spiky bundle. Then $\Gamma(\pi)^{*}$ can be identified with $l^{1}(X)$, the space of summable functions on $X$. A point evaluation $e v_{x} \in \Gamma(\pi)^{*}$ can be interpreted as both a strongly extreme point of $E_{x}^{*} \simeq \mathbb{R}$, and a strongly extreme point of $\Gamma(\pi)^{*}$, but $x$ is not isolated in $X$. The norm-continuity condition on a bundle is thus necessary.

Recall that a Banach space $E$ is said to be strictly convex (= rotund = strictly normed) if and only if whenever $x \neq y \in B(E)$, and $t \in(0,1)$, then $\|t x+(1-t) y\|<1$. That is, proper convex combinations from the unit ball of $E$ stay strictly inside the unit ball. 
Proposition 11. Let $\pi: \mathscr{E} \rightarrow X$ be a bundle, and suppose that $E_{X}$ is strictly convex for each $x \in X$. If $\sigma \in \Gamma(\pi)$ and $\|\sigma(x)\|=1$ for each $x \in X$, then $\sigma \in \operatorname{extr}(B(\Gamma(\pi)))$.

Proof. Suppose that $\sigma=1 / 2 *\left(\tau+\tau^{\prime}\right)$, where $\boldsymbol{\tau} \neq \boldsymbol{\tau}^{\prime} \in B(\Gamma(\pi))$. Then for some $x \in X$, we have $\|\sigma(x)\|=1=\left\|1 / 2 *\left(\tau+\tau^{\prime}\right)(x)\right\|$ and $\tau(x) \neq \tau^{\prime}(x)$. But $1 / 2 *(\tau+$ $\left.\tau^{\prime}\right)(x)$ is a strict convex combination from the unit ball of $E_{x}$, and so by hypothesis $\left\|1 / 2 *\left(\tau+\tau^{\prime}\right)(x)\right\|<1$, a contradiction.

Thus, in the case where each $E_{\chi}$ is strictly convex, we obtain the characterization $\sigma \in$ $B(\Gamma(\pi))$ is an extreme point if and only if $\|\sigma(x)\|=1$ for all $x \in X$. (The converse to the above follows from Proposition 1.) Note that this is the same proof as for $C(X, E)$.

EXAMPLE 12. There is a continuous normed real bundle $\pi: \mathscr{E} \rightarrow X$ and a section $\sigma \in \operatorname{extr}(B(\Gamma(\pi)))$ such that for some $p \in X, \sigma(p) \notin \operatorname{extr} B\left(E_{p}\right)$. Set $E=\mathbb{R}^{2}$ with norm given by $\|\alpha\|_{1}=\left|\alpha_{1}\right|+\left|\alpha_{2}\right|, E_{p}=\mathbb{R}^{2}$ with $\|\alpha\|_{p}=\left(\left|\alpha_{1}\right|^{p}+\left|\alpha_{2}\right|^{p}\right)^{1 / p}, 1 \leq p \leq 2$. Let $X=[1,2]$. For $\alpha \in \mathbb{R}^{2}$, note that $\|\alpha\|_{1} \geq\|\alpha\|_{p} \geq\|\alpha\|_{q} \geq\|\alpha\|_{2}$ when $1 \leq p \leq q \leq 2$. Thus, for $p \geq 1$ and $\alpha \in \mathbb{R}^{2}$ fixed, there exists $c_{p} \geq 1$ such that $c_{p}\|\alpha\|_{p}=\|\alpha\|_{1}$, and $p \mapsto c_{p}$ is continuous (because $p \mapsto\|\alpha\|_{p}=\left(\left|\alpha_{1}\right|^{p}+\left|\alpha_{2}\right|^{p}\right)^{1 / p}$ is continuous).

Suppose now that $\sigma \in C(X, E)$. Then $p \mapsto\|\sigma(p)\|_{p}$ is continuous. For, if $\varepsilon>0$, we can choose $q$ so close to $p$ that both $\left|\|\sigma(p)\|_{p}-\|\sigma(p)\|_{q}\right|<\varepsilon$ and $\|\sigma(p)-\sigma(q)\|_{1}<\varepsilon$. Then

$$
\begin{aligned}
\left|\|\sigma(p)\|_{p}-\|\sigma(q)\|_{q}\right| & \leq\left|\|\sigma(p)\|_{p}-\|\sigma(p)\|_{q}\right|+\left|\|\sigma(p)\|_{q}-\|\sigma(q)\|_{q}\right| \\
& \leq \varepsilon+\|\sigma(p)-\sigma(q)\|_{q} \\
& \leq \varepsilon+\|\sigma(p)-\sigma(q)\|_{1} \\
& <2 \varepsilon .
\end{aligned}
$$

Thus the space of selections $\left\{\sigma: X \rightarrow \dot{\bigcup}\left\{E_{p}: 1 \leq p \leq 2\right\}, \sigma \in C(X, E)\right\}$ is a dense subspace of continuous-normed selections in a bundle $\pi: \mathscr{E}=\dot{\cup}_{p \in X} E_{p} \rightarrow X$. For $\sigma \in C(X, E)$, with norm $\|\sigma\|=\sup \left\{\|\sigma(p)\|_{1}: p \in X\right\}$, we can renorm $\sigma$ by $\|\sigma\|^{\prime}=$ $\sup \left\{\|\sigma(p)\|_{p}: p \in X\right\}$. Then the map $I: C(X, E) \rightarrow \Gamma(\pi),(\sigma,\|\cdot\|) \mapsto\left(\sigma,\|\cdot\|^{\prime}\right)$ is easily checked to be norm-decreasing, with dense range. But since for $\sigma \in C(X, E)$ we also have $\|\sigma\| \leq \sqrt{2}\|\sigma\|^{\prime}$, the range of $I$ is therefore closed, and so there is a topological linear isomorphism of $C(X, E)$ and $\Gamma(\pi)$.

Consider the section $\sigma \in C(X, E)$ given by $\sigma(p) \equiv(1 / 2,1 / 2) /\|(1 / 2,1 / 2)\|_{p}$. Then $\|\sigma(p)\|_{p}=1$ for all $p$; for $1<p \leq 2, \sigma(p)$ is an extreme point of $B\left(E_{p}\right)$; and $\sigma(1)$ is not an extreme point of $B\left(E_{1}\right)$. However, by Proposition $1, \sigma$ is an extreme point of $B(\Gamma(\pi))$.

In several of the cited references, for example [3, 6], complete characterizations of various forms of extreme points are obtained for $B(C(X, E))$ and $B\left(C(X, E)^{*}\right)$. The proofs rely heavily on a complete description of $C(X, E)^{*}$ and also on the constancy of the spaces in which functions take their values. That is, if $\sigma \in C(X, E)$, and if $x, y \in X$, we can talk about closeness of $\sigma(x)$ and $\sigma(y)$ in a quite standard way, using the norm on $E$. Unfortunately, for a bundle $\pi: \mathscr{E} \rightarrow X$ there is in general no nice description of $\Gamma(\pi)^{*}$; likewise, if $\sigma \in \Gamma(\pi)$, and if $x, y \in X$, then $\sigma(x)$ can be "close" to $\sigma(y)$ only in terms of the topology of $\mathscr{E}$, and not necessarily in a metric sense. Intuitively, the 
assumption of norm continuity in the bundle binds fibers together more tightly than norm upper semicontinuity. The separability condition allows for a description of the dual of the section space. Thus, the addition of the conditions of norm continuity and separability of the bundle may eventually allow us to get around some of these difficulties, but it is not immediately apparent how this ought to be done. In general, there appears to the author to be a loose sort of "zero-one" law in effect: when an extremal result for $\Gamma(\pi)$ or $\Gamma(\pi)^{*}$ is proved here, it is arrived at through a tweaking of similar results for spaces $C(X, E)$. On the other hand, many results, which in the $C(X, E)$ case use very strongly either a description of $C(X, E)^{*}$ or a metric notion of nearness, appear in the bundle situation to be nonobvious.

\section{REFERENCES}

[1] E. Behrends, $M$-Structure and the Banach-Stone Theorem, Lecture Notes in Mathematics, vol. 736, Springer-Verlag, Berlin, 1979.

[2] F. Cunningham Jr. and N. M. Roy, Extreme functionals on an upper semicontinuous function space, Proc. Amer. Math. Soc. 42 (1974), 461-465.

[3] P. N. Dowling, Z. Hu, and M. A. Smith, Extremal structure of the unit ball of $C(K, X)$, Banach Spaces (Mérida, 1992), Contemp. Math., vol. 144, American Mathematical Society, Rhode Island, 1993, pp. 81-85.

[4] G. Gierz, Bundles of Topological Vector Spaces and Their Duality, Lecture Notes in Mathematics, vol. 955, Springer-Verlag, Berlin, 1982.

[5]__ Integral representations of linear functionals on function modules, Rocky Mountain J. Math. 17 (1987), no. 3, 545-554.

[6] Z. Hu and M. A. Smith, On the extremal structure of the unit ball of the space $C(K, X)^{*}$, Function Spaces (Edwardsville, Ill, 1994), Lecture Notes in Pure and Appl. Math., vol. 172, Dekker, New York, 1995, pp. 205-222.

[7] _ On the extremal structure of the unit balls of Banach spaces of weakly continuous functions and their duals, Trans. Amer. Math. Soc. 349 (1997), no. 5, 1901-1918.

[8] A. Hulanicki and R. R. Phelps, Some applications of tensor products of partially-ordered linear spaces, J. Functional Analysis 2 (1968), 177-201.

[9] J. W. Kitchen and D. A. Robbins, Tensor products of Banach bundles, Pacific J. Math. 94 (1981), no. 1, 151-169.

[10] G Gel'fand representation of Banach modules, Dissertationes Math. (Rozprawy Mat.) 203 (1982), 47.

[11]___ Integral operators on the section space of a Banach bundle, Int. J. Math. Math. Sci. 16 (1993), no. 3, 449-458.

[12] V. Montesinos, A note on weakly continuous Banach space valued functions, Quaestiones Math. 15 (1992), no. 4, 501-503.

[13] T. S. S. R. K. Rao, Points of weak*-norm continuity in the dual unit ball of injective tensor product spaces, Collect. Math. 50 (1999), no. 3, 269-275.

[14] I. Singer, Linear functionals on the space of continuous mappings of a compact Hausdorff space into a Banach space, Rev. Roumaine Math. Pures Appl. 2 (1957), 301-315 (Russian).

D. A. Robbins: Department of Mathematics, Trinity College, HaRTFord, CT 06106, USA E-mail address: david. robbins@trinco11.edu 


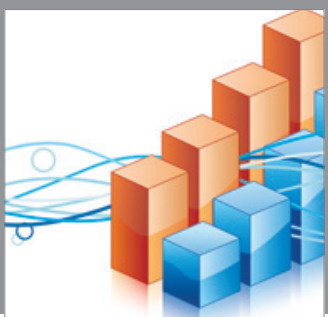

Advances in

Operations Research

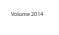

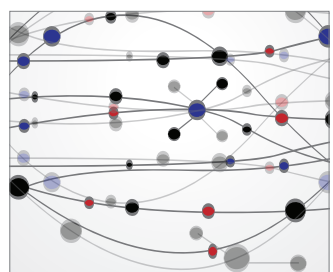

\section{The Scientific} World Journal
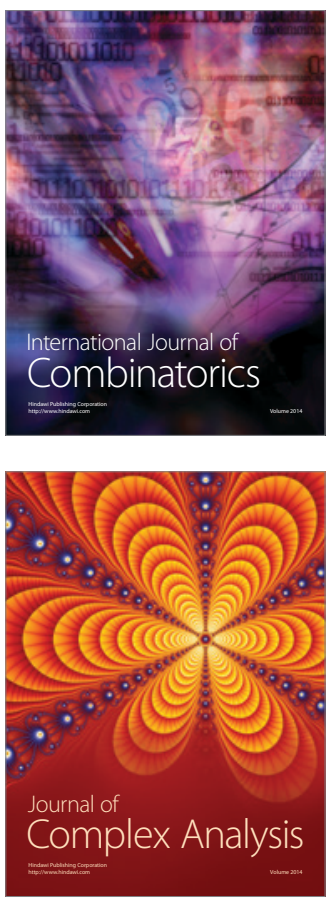

International Journal of

Mathematics and

Mathematical

Sciences
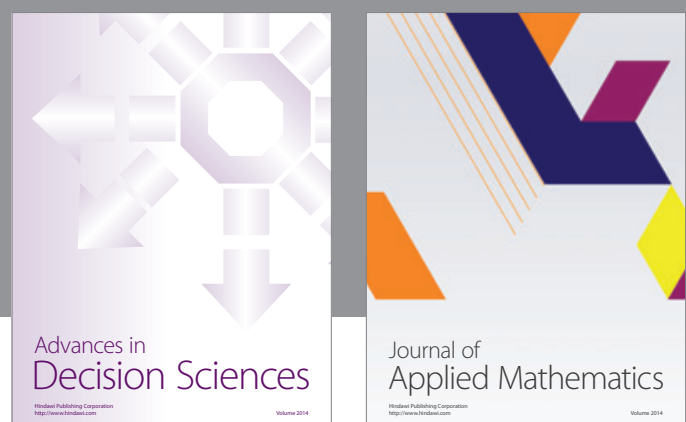

Journal of

Applied Mathematics
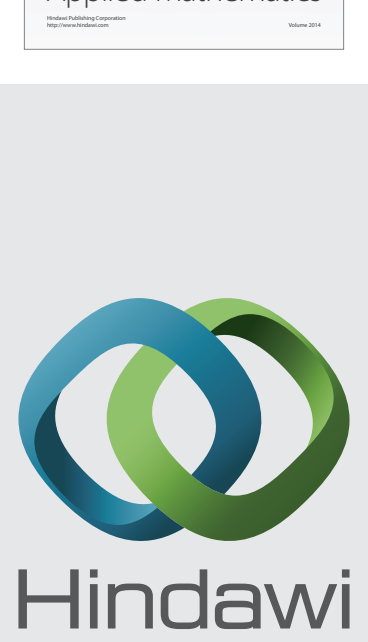

Submit your manuscripts at http://www.hindawi.com
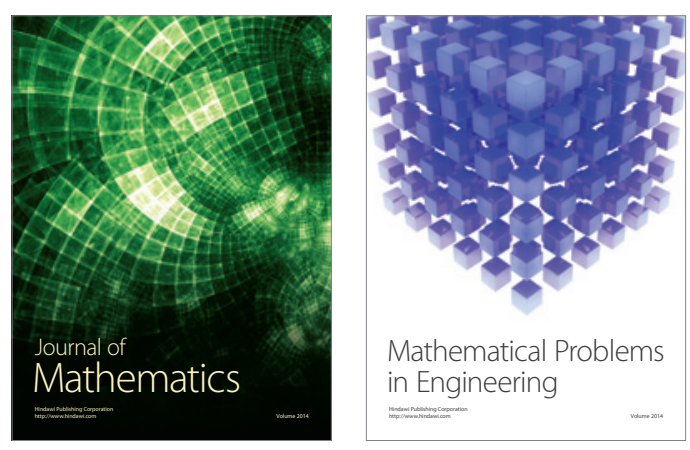

Mathematical Problems in Engineering
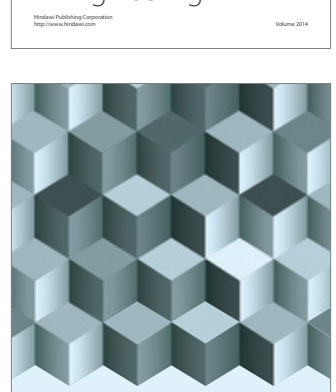

Journal of

Function Spaces
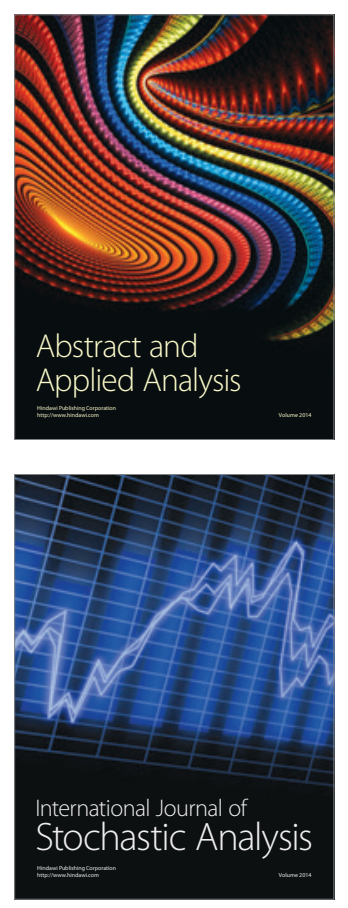

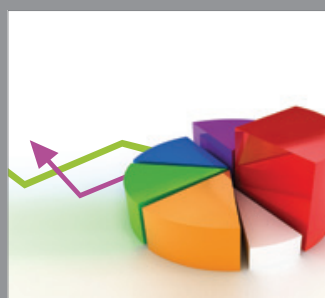

ournal of

Probability and Statistics

Promensencen
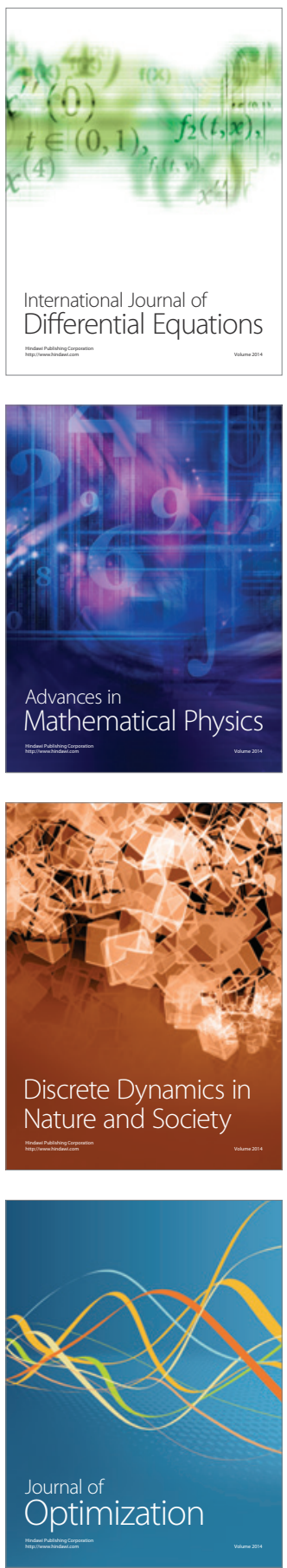